\title{
Assessment and comparison of combined bivariate and AHP models with logistic regression for landslide susceptibility mapping in the Chaharmahal-e-Bakhtiari Province, Iran
}

\begin{abstract}
Landslide is one of the most important natural hazards that make numerous financial damages and life losses each year in the worldwide. Identifying the susceptible areas and prioritizing them in order to provide an efficient susceptibility management is very vital. In current study, a comparative analysis was made between combined bivariate and AHP models (bivariate-AHP) with a logistic regression. At first, landslide inventory map of the study area was prepared using extensive field surveys and aerial photographs interpretation. In the next step, nine landslide causative factors were selected including altitude, slope percentage, slope aspect, lithology, distance from faults, streams and roads, land use, and precipitation which affect occurrence of the landslides in the study area. Subsequently, landslide susceptibility maps were produced using weighted (AHP) bivariate and logistic regression models. Finally, receiver operating characteristics (ROC) curve was used in order to evaluate the prediction capability of the mentioned models for landslide susceptibility mapping. According to the results, the combined bivariate and AHP models provided slightly higher prediction accuracy than logistic regression model. The combined bivariate and AHP, and logistic regression models had the area under the curve (AUC-ROC) values of 0.914, and 0.865 , respectively. The resultant landslide susceptibility maps can be useful in appropriate watershed management practices and for sustainable development in the regions with similar conditions.
\end{abstract}

Keyword: Landslide susceptibility; Combined bivariate and AHP models; Logistic regression; GIS; Iran 
